\title{
Electromagnetic full particle simulation of the electric field structure around the moon and the lunar wake
}

\author{
Shinya Kimura* and Tomoko Nakagawa \\ Tohoku Institute of Technology, 35-1 Yagiyama Kasumi-cho, Taihaku-ku, Sendai, Miyagi 982-8577, Japan
}

(Received April 24, 2007; Revised February 12, 2008; Accepted February 26, 2008; Online published July 4, 2008)

\begin{abstract}
The electric field structure around the moon is studied using a 2-dimensional electromagnetic full particle simulation. By considering absorption of the plasma particles at the surface of the moon, we obtain an intense electric field at the terminator region where the electric field produced by the negatively charged lunar surface and the ambipolar electric field at the wake boundary are in the same direction. The intensity of the electric field is $2.2 E_{0}\left(E_{0}=m_{0} v_{e} \omega_{p} / q_{0}\right)$ at the terminator, corresponding to $3.5 \mathrm{~V} \mathrm{~m}^{-1}$ in the solar wind. It has a large horizontal component due to the potential difference between the negatively charged, antisolarside surface of the moon and the electrically neutral, solar-side surface, even though the emission of photoelectrons are not taken into consideration in this study. The half width of the electric field structure is of the order of Debye shielding length. The electric field at the downstream wake boundary at $x=6.5 R_{L}$ is still as large as $0.1 E_{0} \sim 0.16 \mathrm{~V} \mathrm{~m}-1$, which is strong enough to cause the pitch angle diffusion of the solar-wind electron beam, as is expected in the generation mechanism of the wake-related whistler wave. The ion acceleration occurs in the close vicinity of the moon and can be explained by the acceleration by the electric field produced by the surface charging of the moon. Key words: Lunar wake, electric field, wake potential structure, 2D electromagnetic PIC code.
\end{abstract}

\section{Introduction}

The solar wind interaction with the moon is quite different from that with the Earth. The moon, exposed to the solar wind stream due to the absence of the global magnetic field, absorbs the plasma particles that hit the surface, creating the plasma cavity called the lunar wake on the anti-solar side of the moon (Schubert and Lichtenstein, 1974, and references therein).

The absorption of the solar wind plasma affects the electrostatic potential of the lunar wake and the lunar surface. At the boundary of the lunar wake, the solar wind ions and electrons are thought to rush into the central void region at different speeds. It is essential for the formation of the electric potential structure of the lunar wake that the electron thermal speed is larger than the solar wind bulk speed while the ion thermal speed is smaller than that of the solar wind. Due to the difference of the thermal speeds, the ambipolar electric field is formed at the wake boundary (Samir $e t$ al., 1983). Although no direct measurement of the electric field has been made, ion flows accelerated by the ambipolar electric field inward into the central void were detected by WIND during the passage through the wake (Ogilvie et al., 1996). In the upstream of the wake, WIND and NOZOMI detected enhancements of backstreaming electrons that are supposed to be reflected by the electric field at the wake

\footnotetext{
*Now at NTT Software Corporation, Teisan Kannai Bldg. 4F, 209, Yamasitatyo, Nakaku, Yokohama, Kanagawa 231-8551, Japan.

Copyright (C) The Society of Geomagnetism and Earth, Planetary and Space Sciences (SGEPSS); The Seismological Society of Japan; The Volcanological Society of Japan; The Geodetic Society of Japan; The Japanese Society for Planetary Sciences; TERRAPUB.
}

boundary (Farrell et al., 1996; Futaana et al., 2001).

At the same time, the accumulation of negative charge occurs on the nightside surface of the moon, which produces a significant potential drop. The anti-solar side surface of the moon charges negative because it is hit by the electrons whose thermal speed is larger than the bulk speed of the solar wind. The ions, whose thermal speed is smaller than the solar wind speed, cannot reach the anti-solar side surface of the moon. The negative charging of the shadowed surface of the moon was evidenced by the observation by Lunar Prospector (Halekas et al., 2002). The electric field structure around the moon is largely influenced by the surface charging of the moon. By measuring the energy shift of the electron distribution function obtained from Lunar Prospector, Halekas et al. (2005) estimated the electrostatic potential drops of several hundred volts in the central void of the wake.

The early observations of the lunar wake by Explorer 35 (Lyon et al., 1967; Colburn et al., 1967; Ness et al., 1968) triggered theoretical studies and numerical experiments, many of which aimed at reproducing the largescale structure and magnetohydrodynamic nature of the lunar wake, such as the formation of the cavity, the intensification of the magnetic field in the central void, and the penumbral decrease of the magnetic field (e.g., Whang, 1969; Beers, 1972, 1973; Lipatov, 1976). The main subject of the simulation works shifted to the kinetic effects of the solar wind particles in the wake, since the WIND spacecraft revealed the kinetic nature of the electromagnetic phenomena in the wake, such as counterstreaming ion beams (Ogilvie et al., 1996), a wide range of wave activities (Kellogg et al., 1996), and upstream ULF waves associated with 
backstreaming electrons (Farrell et al., 1996). Farrell et al. (1998) reproduced wakeward ion beams generated by the ambipolar electric field formed at the wake edges, using a simple one-dimensional (1D) electrostatic particle-incell code. Birch and Chapman $(2001,2002)$ showed that the dynamics of the ions are governed by the electrostatic potentials created by electrons, using $1 \frac{1}{2} \mathrm{D}$ and $2 \frac{1}{2} \mathrm{D}$ electromagnetic particle-in-cell codes. Trávníček et al. (2005) carried out a 2-D hybrid simulation with protons described as particles and electrons as massless fluid, in order to study the downstream structure and fluctuations of the lunar wake together with counterstreaming and anisotropic ion distributions.

A GEOTAIL observation of Doppler-shifted whistler waves (Nakagawa et al., 2003) suggested an intense electric field in the vicinity of the moon. The whistler waves were observed to propagate sunward along the magnetic field when the spacecraft far upstream of the moon was magnetically connected with the lunar wake. To generate the whistler wave, the pitch angle of the solar wind electron beam needs to be diffused by an intense electric field of the order of $2.8-4.0 \times 10^{-2} \mathrm{~V} / \mathrm{m}$ at the wake boundary (Nakagawa and Iizima, 2005, 2006). Such an intense ambipolar electric field, if present, would be found in the vicinity of the moon according to the theoretical model (Samir et al., 1983). On the other hand, the electric potential near the moon might be affected by the surface charging of the moon. There has been no observation of the electric field from the spacecraft in the low-altitude wake boundary. There has also been no simulation study that has dealt with the formation of the wake field and the surface charging in a self-consistent manner. Kallio (2005) studied nonaxisymmetric filling of the tail with solar wind plasma by using a 3-D quasi-neutral hybrid model, but the ambipolar electric field was initially given in the model. Guio and Pécseli $(2004,2005)$ investigated ion flow around an obstacle in a streaming plasma with a particle-in-cell simulator, but the net charge density inside the obstacle was fixed to zero in their simulation.

In order to examine the electric field at the wake boundary close to the moon, we need to calculate the ambipolar electric field at the wake boundary and the surface charging simultaneously in a self-consistent manner. This paper reports the results from a 2-D full particle electromagnetic simulation in the vicinity of the moon.

\section{Numerical Simulation}

\subsection{Two-dimensional electromagnetic PIC simulation}

To deal with the surface charging, the electrons must be treated as particles. The round shape of the moon should be reproduced at the very least in a 2-D space. A 2-D, fullparticle electromagnetic code (Birdsall and Langdon, 1985) is used in this study. An electrostatic code is also used for comparison and confirmation of the structure. We had to give up on a 3-D simulation because it is more important to have large number of mesh points under the limitation of the memory resources so that the spatial separation is small enough with respect to the Debye shielding length.

The effects of photoelectrons and the crustal magnetic fields are not included for the purpose of simplicity, al- though they would be important.

The effect of the interplanetary magnetic field is another interesting subject, but here in this paper, we limit ourselves to the cases with no background magnetic field to concentrate the surface charging effect and the ambipolar electric field at the edges of the wake.

\subsection{Equations}

The equation of motion for the ions and electrons

$$
m \frac{d \mathbf{v}}{d t}=q\left(\mathbf{E}+\frac{\mathbf{v}}{c} \times \mathbf{B}\right)
$$

is solved for every time step to obtain the position and the velocity of the particles, from which the charge density

$$
\rho=\Sigma q
$$

as well as the current density

$$
\mathbf{J}=\Sigma q \mathbf{v}
$$

is calculated on the spatial grids where the electric field $\mathbf{E}$ and magnetic field $\mathbf{B}$ are defined. Here, $m, q$, and $\mathbf{v}$ are mass, charge, and velocity of each particles, and $c$ is the speed of light, respectively. Normalization of the variables is given in Appendix A. The Heaviside-Lorentz system is employed in this paper. The Maxwell's equations

$$
\begin{aligned}
& \frac{\partial \mathbf{E}}{\partial t}=c \nabla \times \mathbf{B}-\mathbf{J} \\
& \frac{\partial \mathbf{B}}{\partial t}=-c \nabla \times \mathbf{E}
\end{aligned}
$$

are solved using the FDTD method.

In the particle-in-cell simulations, it is often the case that Gauss' law

$$
\nabla \cdot \mathbf{E}=\rho
$$

is not applicable because of microscopic inconsistencies between $\mathbf{J}$ and $\rho$ due to use of the mesh and weights (chapter 15-6 of Birdsall and Langdon, 1985). A correction term $\delta \phi$ is introduced so that $(\mathbf{E}-\nabla \delta \phi)$ in place of $\mathbf{E}$ should satisfy the Gauss' law as

$$
\nabla \cdot(\mathbf{E}-\nabla \delta \phi)=\rho .
$$

The correction $\delta \phi$ is obtained by solving the Fourier transform of Eq. (7) and then inverse transforming the Fourier components. This procedure requires a periodic boundary condition for the electric field.

\subsection{Configuration of the simulation box}

In order that the periodic boundary condition does not affect the electric field of the region of interest, we need a large simulation box. In this study, we employ a $20 R_{L} \times 20 R_{L}$ simulation box $\left(-5 R_{L}<x<15 R_{L}\right.$, $-10 R_{L}<y<10 R_{L}$ ), which is large enough with respect to the area of interest within a few lunar radii $\left(R_{L}\right)$ from the moon. The box is divided into $256 \times 256$ cells, each of which contains, on average, more than 120 particles for each species. Thus, the solar wind plasma around the moon is represented by $7.86 \times 10^{6}$ ions and as many electrons.

The grid size is derived from the system length $L=$ $20 R_{L}$ divided by the number of grids $N_{g}=256$ as

$$
\Delta x=\Delta y=\frac{L}{N_{g}}=\frac{20 R_{L}}{256} \text {. }
$$




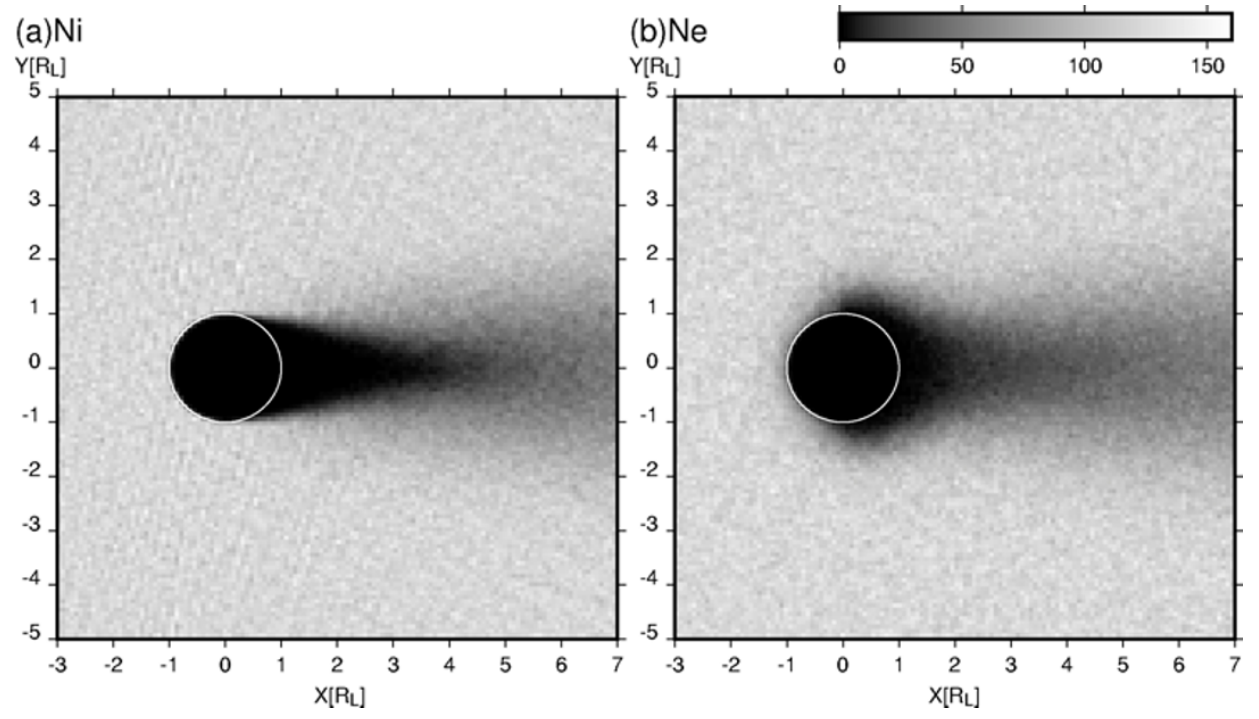

Fig. 1. Two-dimensional plots of (a) the proton density $n_{i}$ and (b) the electron density $n_{e}$ around the moon (outlined with a white circle) for the simulation run \#2 at $t=9.4 \times 10^{2} \omega_{p}^{-1}$ (60000 steps). The solar wind flows from the left to the right.

Table 1. Choice of parameters.

\begin{tabular}{cccl}
\hline Run & $R_{L} / \lambda_{D}$ & $\Delta x / \lambda_{D}$ & $v_{e} / c$ \\
\hline$\# 1$ & 2 & $1 / 6.4$ & 0.1 \\
$\# 2$ & 4 & $1 / 3.2$ & 0.1 \\
$\# 3$ & 8 & $1 / 1.6$ & 0.05 \\
\hline
\end{tabular}

As we deal with the electric field produced by the surface charging, the spatial grid size $\Delta x$ and $\Delta y$ needs to be small enough with respect to the Debye length

$$
\Delta x<\lambda_{D}
$$

where $\lambda_{D}$ is the Debye length

$$
\lambda_{D} \equiv \frac{v_{e}}{\sqrt{2} \omega_{p}}
$$

and $\omega_{p}=\sqrt{n q^{2} / m_{e}}$ is the plasma frequency. From Eqs. (8) and (9), it turns out that the scale size of the obstacle with respect to the Debye length $R_{L} / \lambda_{D}$ has to be smaller than 12.8. On the other hand, the Debye length, which is typically $10-100 \mathrm{~m}$ in the solar wind, is much smaller than the lunar radius $R_{L}$, consequently the ratio $R_{L} / \lambda_{D}$ should be greater than unity. In the range $1<R_{L} / \lambda_{D}<12.8$, we employ $R_{L} / \lambda_{D}=2,4$ or 8 , each of which corresponds to $\Delta x / \lambda_{D}=0.16,0.31$, or 0.63 , respectively. Table 1 summarizes the parameters for each simulation runs.

The time step $\Delta t=\left(1-10^{-3}\right) \Delta x / \sqrt{2} c$ is set so that it satisfies the Courant condition

$$
\frac{1}{(c \Delta t)^{2}} \geq \frac{1}{(\Delta x)^{2}}+\frac{1}{(\Delta y)^{2}} .
$$

\subsection{Initial and boundary conditions}

At the beginning of the simulation, the particles are uniformly distributed over the entire configuration space except for the inside of the moon. The initial velocity distri- butions are shifted Maxwellian

$$
f_{s}(\mathbf{v})=\frac{n_{s}}{\pi^{\frac{3}{2}} v_{s}^{3}} \exp \left(-\frac{\left(v_{x}-v_{s w}\right)^{2}+v_{y}^{2}+v_{z}^{2}}{v_{s}^{2}}\right),
$$

where $v_{s w}$ is the solar wind speed and $v_{s}$ is the thermal speed for each species $s$ ( $s=i$ for ions and $e$ for electrons). As the simulation starts, the plasmas flow down the simulation domain due to the antisunward bulk velocity $\left(v_{s w}, 0,0\right)$, forming the plasma cavity behind the moon. In this simulation, we use the solar wind speed $v_{s w}=0.25 v_{e}$, where $v_{e}$ is the electron thermal speed. To reproduce the configuration of the lunar wake, the ion thermal speed $v_{i}=3.125 \times 10^{-2} v_{e}$ is chosen so that the ratio $v_{i}: v_{s w}$ would be 1:8 as observed in space.

Since we start with uniformly distributed plasma, we have to wait until the solar wind plasma flows down the simulation box and the plasma void forms in the umbral region behind the moon. To reduce the time for the calculation, we use the solar wind speed $v_{s w}=0.025 c$ together with the electron thermal speed $v_{e}=0.1 c$, both of which are larger than those of the real solar wind. On the surface charging and the wake formation, there would be no impact of the ratios of the solar wind velocity and the electron thermal speed with respect to the speed of light, as far as the result is interpreted in terms of $v_{e}, v_{i}$, and $v_{s w}$.

The boundary condition for the particles is periodic in the $y$ direction. A particle that crosses $y=10 R_{L}$ is re-entered into the simulation domain from $y=-10 R_{L}$, and vice versa. In the $x$ direction, we chose a nonperiodic condition with unperturbed solar wind at the upstream boundary and the perturbed plasma at the downstream end.

\subsection{Injection and removal of the particles}

A particle that crosses the downstream end $x=15 R_{L}$ is removed from the simulation domain. The particles that collide the moon are also removed after they give their electric charge to the moon. On average, $n L v_{s w} \Delta t$ ions are lost every time step $\Delta t$ at the downstream end or at the lunar surface, where $n$ is the average ion density given by 
$N_{p} / L^{2}$ in 2-D space, $N_{p}$ is the total number of ions within the simulation box, and $L=20 R_{L}$ is the system length. To compensate for the loss, as many ions are injected from the upstream boundary $\left(x=-5 R_{L}\right)$ of the simulation domain. The electrons, whose thermal speed is higher than that of the ions, collide with the moon more frequently than the ions and are removed from the simulation box. The injection of electrons is controlled in such a way that the total number of electrons flowing in the simulation box is equal to that of ions.

The velocity distribution of the particles to be injected is given as

$$
F(\mathbf{v})=\left|v_{x}\right| f_{s}(\mathbf{v})
$$

so that the distribution would become Maxwellian in the simulation box after the injection. The position of the injected particle is nearly uniformly distributed on the boundary but with significant random perturbations to avoid some artificial noize.

\subsection{Surface charging of the moon}

The electric charge given by the particles onto the lunar surface are fixed at the position they collide, not moving freely on the surface, as the electric conductivity of the lunar surface is small. Once the simulation starts, the electric charge of the solar wind plasma begins to accumulate on the surface of the moon. The downstreamside surface (nearly nightside surface), hit by only electrons, charges negative. The upstreamside (nearly dayside) surface, hit by both ions and electrons, remains electrically neutral. The positive and negative charges are compiled at each position on the lunar surface and then added to the charge density $\rho$ in Eq. (2) from which the electric field is calculated. Accordingly, as the electric charges accumulate, they produce the electric field which expels newly incident particles. Asymptotically, the surface charge and electric potential became nearly constant when the solar wind plasma swept the simulation domain about 4 times $\left(t=9.4 \times 10^{2} \omega_{p}^{-1}\right)$.

\section{Results}

\subsection{Electric potential around the moon}

Figure 1 shows the proton and electron densities around the moon for the simulation run \#2. The ion void formed behind the moon is the lunar wake. The ions cannot reach the nightside surface of the moon because their thermal speed is smaller than the bulk velocity of the solar wind. The number density of the electrons is also depleted, but not so much as that of the ions. The electrons can reach the nightside of the moon, as the thermal speed is fourfold larger than the solar wind speed.

Figure 2 shows the electric potential $\phi$ around the moon for the same simulation run as in Fig. 1. Consistent with the ion depletion region in Fig. 1(a), a negative potential structure is created in the umbra behind the moon. It is also recognized that the largest potential drop outlines the nightside surface of the moon. This is believed to be caused by the negative charge on the downstreamside surface of the moon where only the electrons can get to. The magnitude of the potential drop caused by the surface charging is larger than that of the downstream wake.

Figure 3 shows the electric potential $\phi$ at the terminator plotted against the distance along the $y$ axis of the simula-

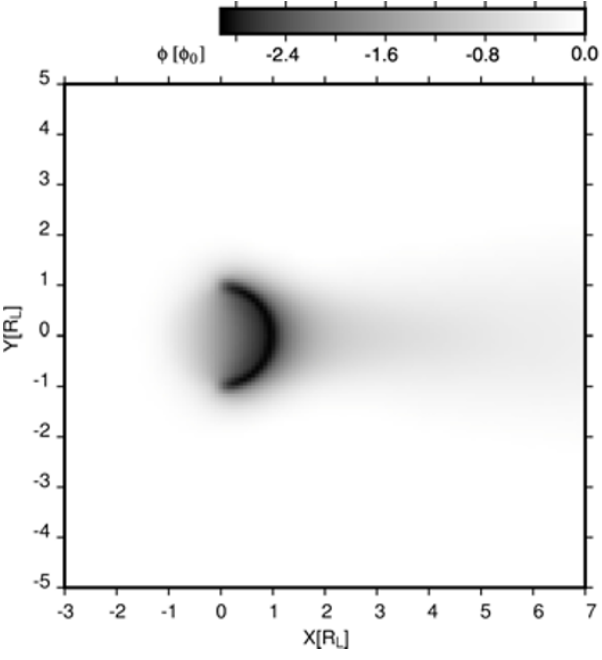

Fig. 2. A gray scale map of the electric potential $\phi$ around the moon for the simulation run \#2. The dark color indicates the negative potential. The negative potential is recognized in the central void of the lunar wake and on the antisolarside surface of the moon.
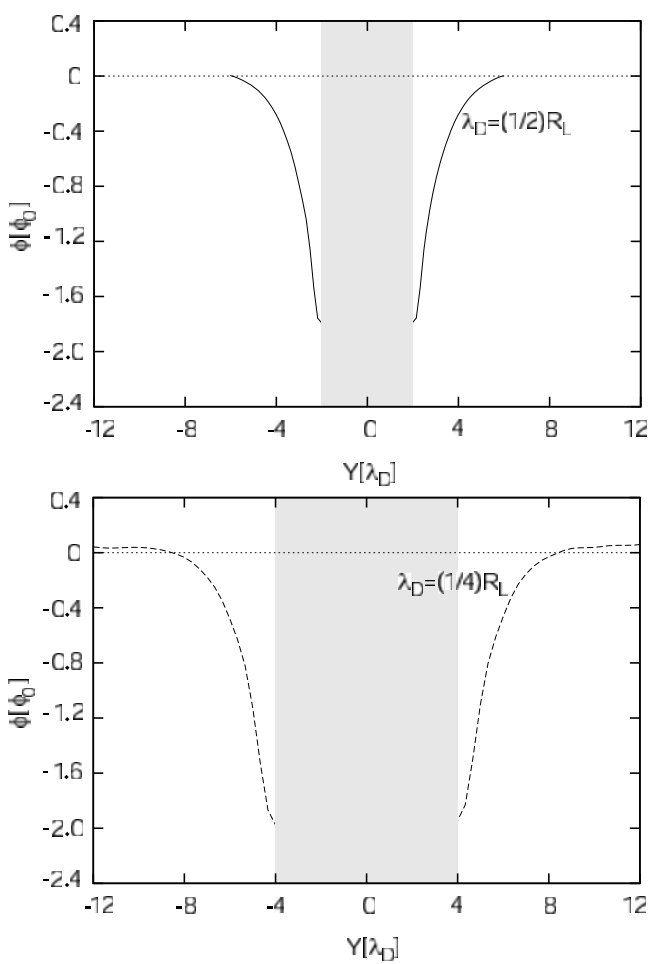

Fig. 3. The electric potential $\phi$ plotted against the distance along the $y$ axis of the simulation box at $x=0$ for the simulation run \#1 (upper panel) and \#2 (lower panel). The normalizing factor for the electric potential is $\phi_{0} \equiv m_{0} v_{e}^{2} / q_{0}$. The shaded area $|y|<1 R_{L}$ indicates the body of the moon.

tion box at $x=0$. At the terminator $\left(y= \pm 1 R_{L}\right)$, the potential drop is as large as $-2 \phi_{0}$, where $\phi_{0} \equiv m_{0} v_{e}^{2} / q_{0}$. Substituting the thermal energy of the solar wind electrons 15$20 \mathrm{eV}$ at the time of detection of the wake-related whistler waves (Nakagawa et al., 2003), we can estimate the magnitude of the potential drop at the terminator to be $60-80 \mathrm{~V}$. It is comparable to the potential difference $-48 \pm 15 \mathrm{~V}$ between the lunar surface and the Lunar Prospector at the altitude of $20-40 \mathrm{~km}$ (Halekas et al., 2002), or the potential 


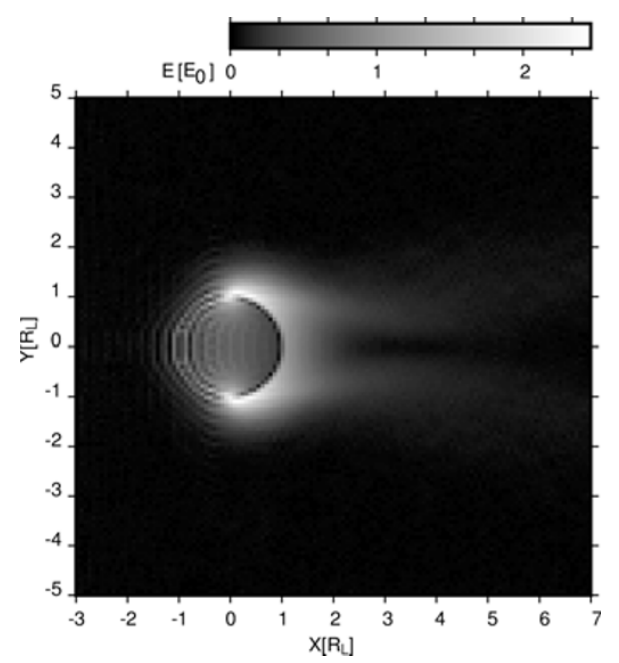

Fig. 4. Magnitude of the electric field obtained from the simulation run \#2 at $t=9.4 \times 10^{2} \omega_{p}^{-1}$. The faint electric field is recognized at the boundary layers of the lunar wake behind the moon. The intense electric field appears at the terminator on the boundary between the electrically neutral dayside surface and negatively charged nightside surface.

of $-442 \mathrm{~V}$ estimated from the WIND observation of the energy of the backstreaming electrons (Farrell, 1996). It is also comparable to the floating potential

$$
\phi=\frac{\phi_{0}}{2} \ln \left\{\left(\frac{m_{e}}{m_{i}}\right)^{\frac{1}{2}}\left(\frac{T_{i}}{T_{e}}\right)^{\frac{1}{2}}\right\}
$$

of an artificial satellite in the solar wind plasma (see, Fahleson, 1967). If we assume $T_{i} \sim T_{e}$, Eq. (14) leads to $\phi \sim-1.9 \phi_{0}$.

The half width of the potential structure is nearly the same as the Debye length.

\subsection{Electric field structure}

Figure 4 shows a gray scale plot of the magnitude of the electric field around the moon. A faint electric field at the wake boundary extends up to $6 R_{L}$ downstream of the moon. The most intense field $E \sim 2.2 E_{0}$, where $E_{0} \equiv m_{0} v_{e} \omega_{p} / q_{0}$, is found at the terminator $\left(x=0\right.$ and $\left.y= \pm 1 R_{L}\right)$ as the result of the surface charging of the moon and the local violation of the charge neutrality. The magnitude $2.2 E_{0}$ of the electric field corresponds to $3.5 \mathrm{~V} \mathrm{~m}^{-1}$ in the solar wind, since the normalizing factor $E_{0}$ is about $1.6 \mathrm{~V} \mathrm{~m}^{-1}$ according to the GEOTAIL observation during the period of the wake-associated whistler waves (Nakagawa et al., 2003). The electric field is strong enough to cause the pitch angle diffusion of the solar wind electron beam through the electric field drift as proposed by Nakagawa and Iizima (2006).

Figure 5 shows the direction of the electric field around the moon. The electric field vectors just antisolarside of the moon are perpendicular to the lunar surface, indicating that they are produced by the surface charging. At the terminator, the electric field has a significant component parallel to the surface because the intense electric field is produced by the potential difference between the negatively-charged, antisolarside surface and the neutral, sunward-side surface. In addition, in the vicinity of the terminator, the ambipolar electric field at the wake boundary is nearly in the same

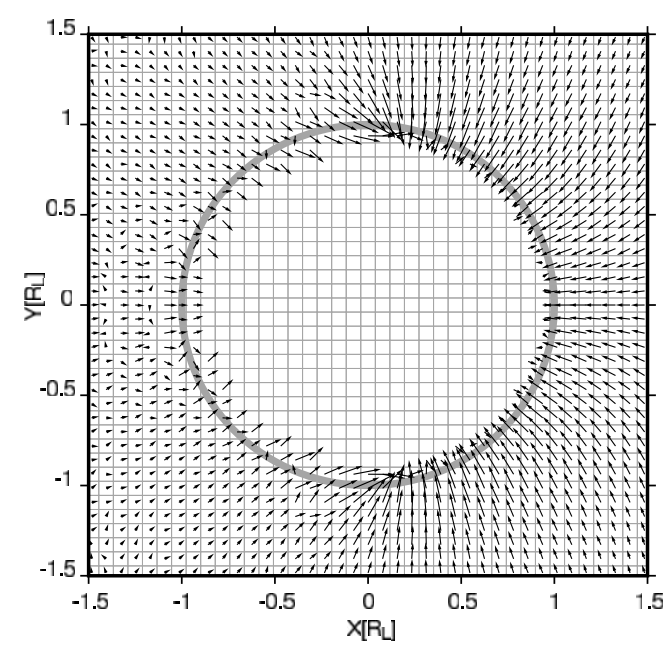

Fig. 5. Direction of the electric field (arrows) around the moon. A gray circle outlines the body of the moon. The solar wind flows from the left to the right. The result of the simulation run \#2.

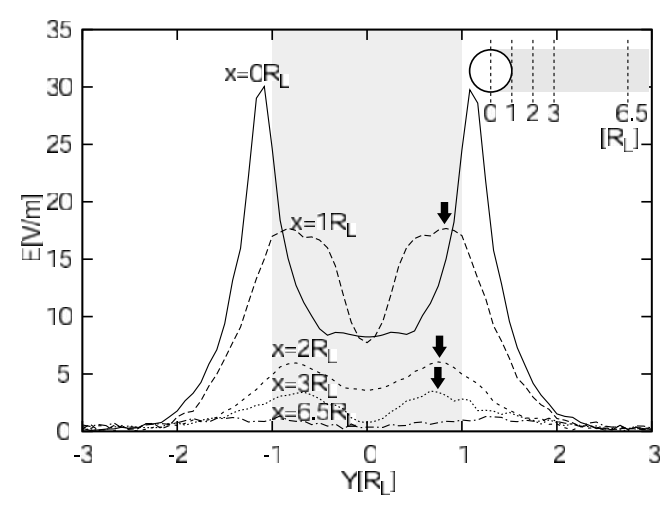

Fig. 6. Magnitude of the electric field plotted against the distance in the $y$ direction in the simulation box at several distances from the moon, $x=0,1 R_{L}, 2 R_{L}, 3 R_{L}$ and $6.5 R_{L}$. The normalizing factor for the electric field magnitude is $E_{0}=m_{0} v_{e} \omega_{p} / q_{0}$. The most intense electric field appears at the terminator $\left(x=0, y= \pm 1 R_{L}\right)$. It is by two to sevenfold lager than that of the peaks (indicated by arrows) of the downstream electric fields. The shaded area $|y|<1 R_{L}$ for the curve $x=0$ should be ignored as it is inside of the moon. The result of the simulation run $\# 2$.

direction as that caused by the surface charging.

The ambipolar electric field at the wake boundary is clearer when it is plotted against the distance in the $y$ direction. Figure 6 shows the magnitude of the electric field at several distances from the moon, $x=0,1 R_{L}, 2 R_{L}, 3 R_{L}$ and $6.5 R_{L}$. The most intense electric field appears at the terminator $\left(x=0, y= \pm 1 R_{L}\right)$. The half width of the electric field is of the order of the Debye length.

The electric fields at the boundaries of the downstream wake at $x=1 R_{L}-3 R_{L}$ is by two to sevenfold smaller than the electric field at the terminator. At $6.5 R_{L}$ downstream, the electric field at the wake boundary is about $0.1 E_{0}$. It is 25-fold smaller than that at the terminator, but it corresponds to $0.16 \mathrm{~V} \mathrm{~m}^{-1}$ in the real solar wind environment, which is again large enough to cause the pitch angle diffusion (Nakagawa and Iizima, 2006).

The position of the peaks of the electric field $y=$ $\pm 0.83 R_{L}$ for $x=1 R_{L}, y= \pm 0.75 R_{L}$ for $x=2 R_{L}$, and 

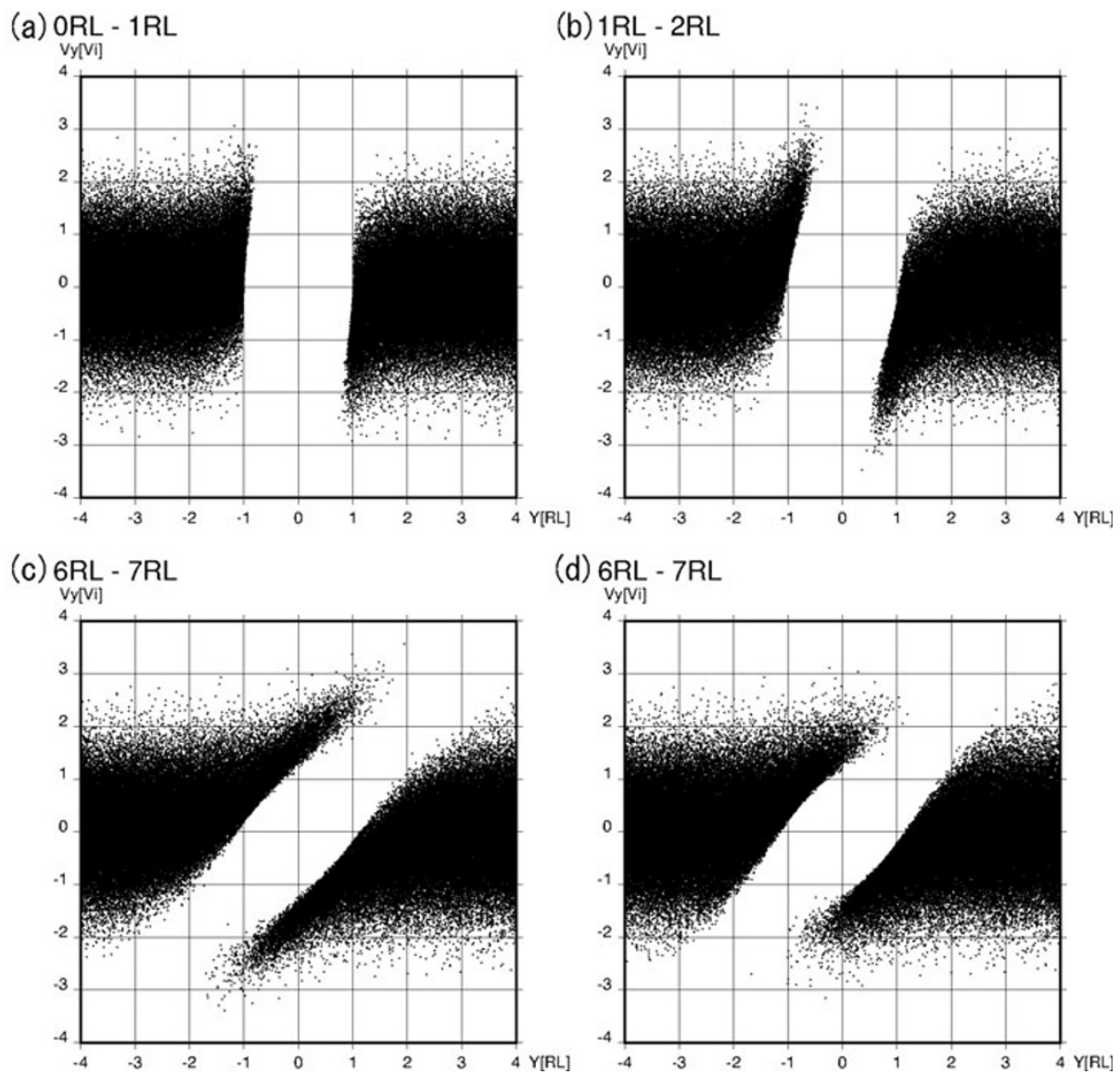

(d) $6 R L-7 R L$

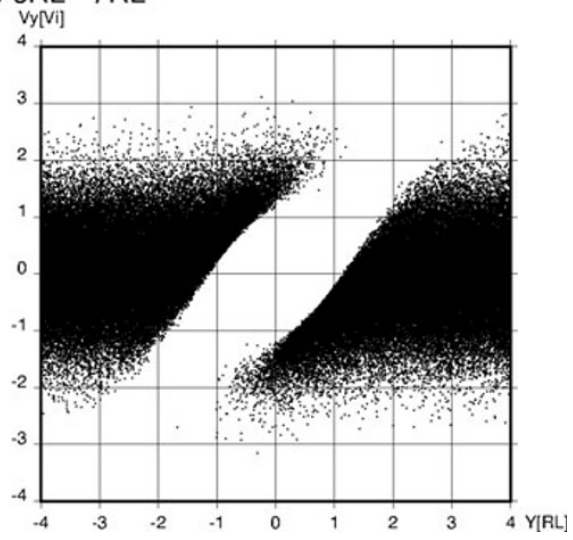

Fig. 7. Distribution of the transverse velocity component $v_{y}$ of ions normalized by the ion thermal speed $v_{i}$ plotted against the distance $y$ from the axis of the void at several distances $x$ from the moon (a) $0<x<1 R_{L}$, (b) $1 R_{L}<x<2 R_{L}$, and (c) $6 R_{L}<x<7 R_{L}$. These are the results for simulation run \#2. (d) Result from the simulation run in which the surface charging of the moon is ignored.

$y= \pm 0.66 R_{L}$ for $x=3 R_{L}$, gets closer to the central axis of the wake as the distance $x$ become larger, in accordance with the ion entry into the central void.

\subsection{Velocity distribution of ions}

One of the advantages of the full-particle simulation is that we can examine the velocity distribution of the particles. In the lunar wake, it is said that the ions are accelerated by the ambipolar electric field toward the center of the void, forming a counterstreaming ion beam (Ogilvie et al., 1996). In the absence of the background magnetic field, the acceleration is expected to be in the $y$ direction. Figure 7 shows the velocity component $v_{y}$ of each ion plotted against the distance in the $y$ direction at several distances $x$ from the moon. Figure 7(a) shows the velocity distribution in the close vicinity of the moon, $0<x<1 R_{L}$. The blank area $-1 R_{L}<y<1 R_{L}$ is the inside of the moon or the umbra behind the moon. The outside $\left(y<-1 R_{L}\right.$ or $y>1 R_{L}$ ) are the solar wind. The velocity is normalized by the ion thermal speed $v_{i}$. Most of the ions are distributed within $\left|v_{y} / v_{i}\right|<2$. It is recognized that only the high-speed ions near the wake boundary $\left(v_{y} / v_{i} \sim 2\right.$ at $y \sim-1 R_{L}$, or $v_{y} / v_{i} \sim-2$ at $y \sim 1 R_{L}$ ) are entering the plasma void. Figure 7(b) shows the region a little downstream $1 R_{L}<$ $x<2 R_{L}$. Fast ions with $v_{y} / v_{i} \sim \pm 2$ enters farther into the void, and the slower ions with $v_{y} / v_{i} \sim \pm 1$ also begin to enter the void. Most of the solar wind ions are distributed within $\left|v_{y} / v_{i}\right|<2$, while at the front line $y \sim \pm 0.7 R_{L}$, the ions are accelerated inward up to $\left|v_{y} / v_{i}\right| \sim 2.5$. This is supposed to be due to the electric field produced by the surface charging, because the high speed is obtained in the very vicinity of the moon. Figure 7 (c) shows the ion distribution far downstream $6 R_{L}<x<7 R_{L}$, where ions form a bi-streaming beam flowing into the center of the void. The fastest ions have reached the other side of the wake structure. The speed of the highest energy component is nearly equal to that in Fig. 7(b), indicating that the acceleration of the high-energy component occurs within $2 R_{L}$ from the moon.

Figure 7(d) shows the result of a comparative simulation in which no surface charging of the moon is considered. In the absence of the surface charging, the ions are distributed mostly within $\left|v_{y} / v_{i}\right|<2$ at $6 R_{L}<x<7 R_{L}$, showing no signature of acceleration. The absence of high-energy ions that would exceed $\left|v_{y} / v_{i}\right|=2$ is significant when compared with Fig. 7(c). Here we conclude that the ion acceleration in the present simulation is mostly due to the surface charging of the moon rather than the ambipolar electric field at the wake boundary.

\subsection{Dependence on the size of the obstacle}

Figure 8 shows the electric field at the terminator for different ratios of $R_{L} / \lambda_{D}$. The electric field is smaller for a larger obstacle with a thicker layer of electric field because the gradient of an electric potential around a charged body is large for a small body and small for a large sphere. The de- 

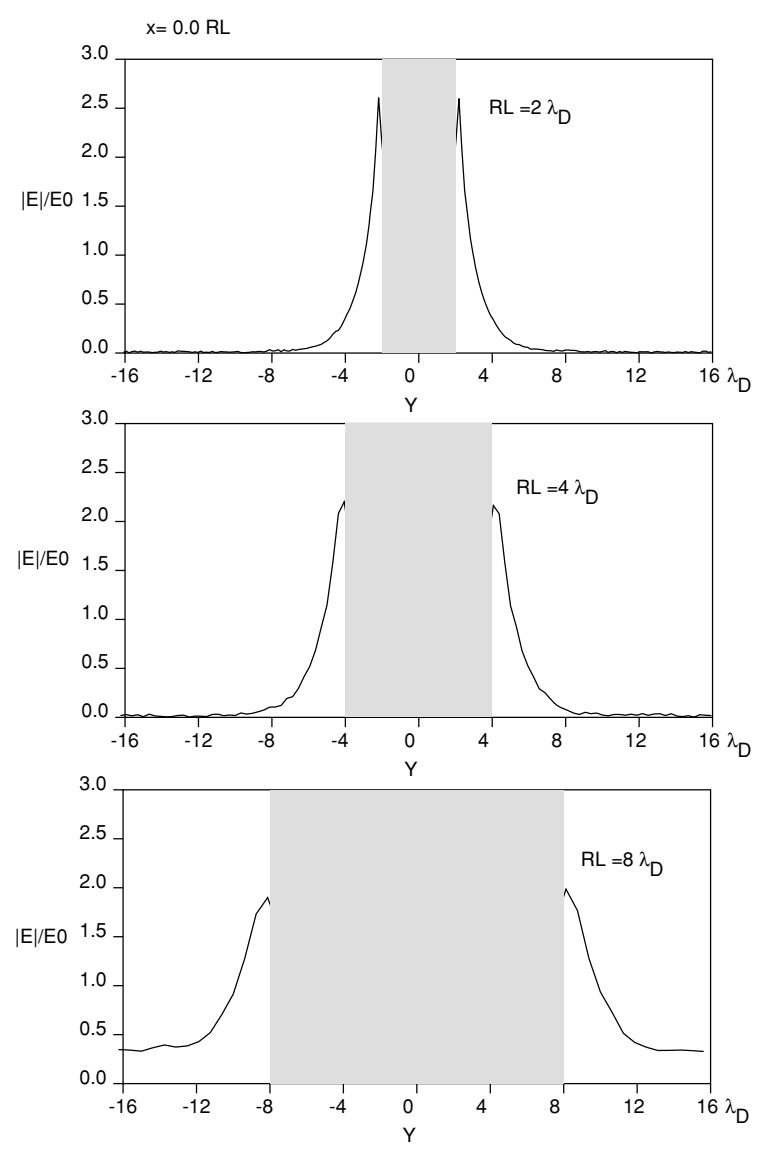

Fig. 8. Electric field at the terminator plotted against the distance along the $y$ axis of the simulation box at $x=0$ for simulation run \#1 (upper panel), \#2 (middle panel) and \#3 (lower panel). The normalizing factor for the electric field magnitude is $E_{0}=m_{0} v_{e} \omega_{p} / q_{0}$. The shaded area $|y|<1 R_{L}$ indicates the inside of the body of the moon.

crease in the magnitude of the electric field becomes slower as the radius of the obstacle becomes larger. It is the same as the electric field structure, which becomes rather 1-D as the charged obstacle become larger with respect to the Debye length.

Figure 9 shows the electric field intensity of the downstream wake for different sizes of the obstacle. The dependence of the electric field intensity on the size of the obstacle diminishes as the distance from the moon increases. At a larger distance from the moon $\left(x=6.5 R_{L}\right)$, the peak intensity of the electric field at the wake boundary is about $0.1 E_{0}$ for all the cases.

\section{Discussion}

The results of the present simulation are summarized as follows:

i) The electric potential structure around the moon consists of the potential drop extending downstream in the lunar wake produced by the negative excess of electric charge in the void, and the potential drop caused by the accumulation of the negative charge on the antisolarside surface of the moon.

ii) The magnitude of the potential drop caused by the surface charging is larger than that of the downstream wake.
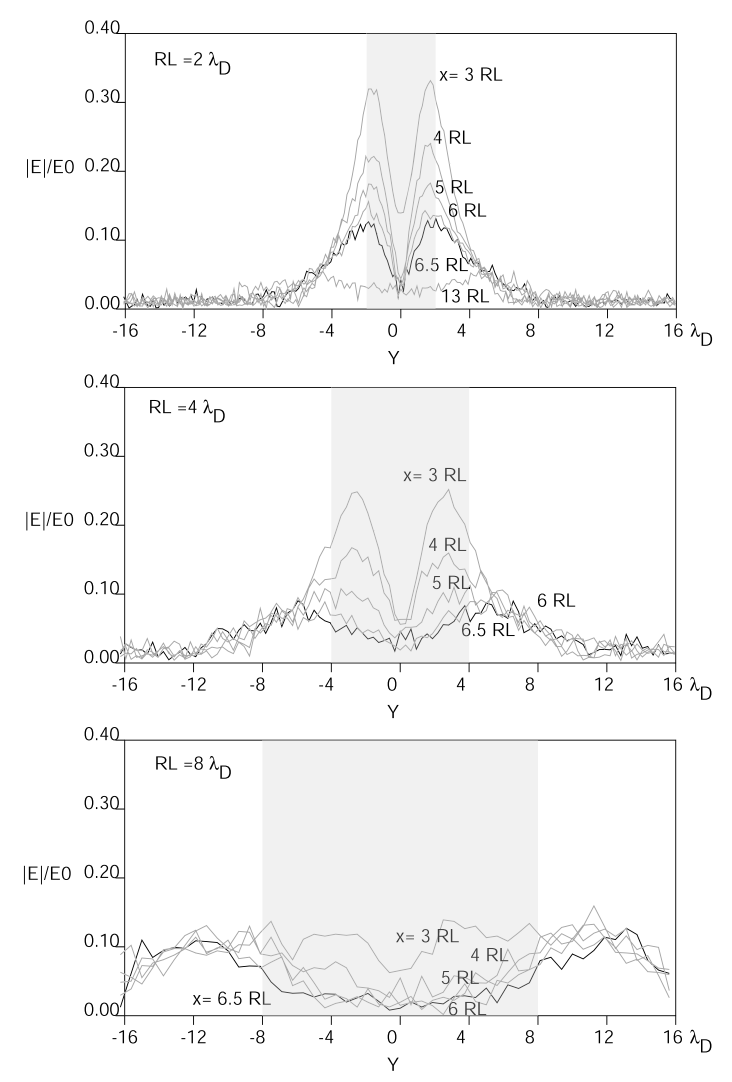

Fig. 9. Electric field in the downstream wake plotted against the distance along the $y$ axis. From top to bottom: Run \#1 $\left(R_{L}=2 \lambda_{D}\right)$, Run \#2 $\left(R_{L}=4 \lambda_{D}\right)$, and Run \#3 $\left(R_{L}=8 \lambda_{D}\right)$. The shaded area indicates the size of the moon.

iii) The largest potential drop $-2 \phi_{0}\left(\phi_{0} \equiv m_{0} v_{e}^{2} / q_{0}\right)$ found at the terminator corresponds to about -60 to $-80 \mathrm{~V}$ in the real solar wind. This is as large as that expected from the theory of surface charging of a conducting satellite immersed in a plasma.

iv) The most intense field 2.2 $E_{0}\left(E_{0} \equiv m_{0} v_{e} \omega_{p} / q_{0}\right)$ is found at the terminator.

v) The electric field has a significant component parallel to the surface at the terminator, while it is orthogonal to the lunar surface in the central void.

vi) The half width of the layer of the electric field is of the order of the Debye shielding length.

vii) The electric field at the downstream wake boundary at $x=6.5 R_{L}$ is still as large as $0.1 E_{0} \sim 0.16 \mathrm{~V} \mathrm{~m}^{-1}$.

viii) The ions are accelerated inward of the wake up to $3 v_{i}$ within $2 R_{L}$ of the moon. The acceleration is not observed in the absence of the surface charging.

ix) The electric field at the terminator is more intense for a smaller obstacle. This dependence on the size of the obstacle is less prominent for the ambipolar electric field at the downstream wake boundary.

The electric field, both at the terminator or the downstream wake boundary, is strong enough to cause the pitch angle diffusion of the solar-wind electron beam through the electric field drift, as proposed by Nakagawa and Iizima (2006).

In the present simulation, there is a limitation of the obstacle size, but the result gives significant estimates of the 
electric field structure around the moon. The result of this study have shown that the electric potential and the electric field in the close vicinity of the moon are essentially the same as those of the conducting satellite in a plasma (Farleson, 1967), except for the potential difference between the neutral, solar-side surface and the negatively-charged, antisolarside surface at the terminator. The intensity of the electric field at the terminator in the real solar wind $\left(R_{L} / \lambda_{D} \sim 10^{4}-10^{5}\right)$ would be smaller than that calculated for $R_{L} / \lambda_{D}=8$ in this simulation; however, the dependence on the ratio gets smaller as the ratio gets larger because the electric potential structure approaches a 1-D approximation of the electric field structure.

The electric field produced by the surface charging extends as far as the Debye shielding length. It is of the order of 10-100 $\mathrm{m}$ in the average solar wind, but it should be noted that it possibly becomes as large as $\sim 1 \mathrm{~km}$ in the wake where the plasma density is depressed (Halekas et al., 2003).

The present simulation is carried out by the electromagnetic code, however, the light speed is physically unimportant in the discussion so far. It will become important when the generation of the whistler mode waves will be discussed in the electric field around the moon produced in this simulation. The result of the electrostatic simulations are essentially the same as those of the electromagnetic simulations.

In this paper, we limit ourselves to the cases of no background magnetic field in order to concentrate on the surface charging and the ambipolar electric field at the edges of the wake. It is expected that the motion of the plasma particles that rush into the void would be affected by the background magnetic field, as observed by WIND (Ogilvie et al., 1996), if the magnitude is strong enough. The case of a weak magnetic field for which the Larmour radius is larger than the size of the obstacle is nearly the same for the case of no background magnetic field, although it is not shown in this paper. The term of the magnetic field is left in the equations for further investigation of the effect of the magnetic field.

We did not consider the effect of dielectric polarization of the lunar material. It seems likely that the electric field due to the surface charge causes dielectric polarization of the surface of the moon. The dielectric polarization works as if the charge density on the surface is reduced. It would decrease the gradient of the electric potential and weaken the electric field outside of the moon.

\section{Conclusion}

In the vicinity of the moon, an intense electric field is produced by the negative charging of the downstreamside surface of the moon and the negative excess of charge in the central void of the wake. The electric field is largest at the terminator, where both of these work in the same direction. The magnitude is large enough to cause the pitch angle diffusion of the solar wind electron beam, which is expected in the generation of the wake-associated whistler wave.

Acknowledgments. The authors are grateful to T. Hada and $\mathrm{S}$. Matsukiyo for valuable comments and suggestions. The authors are also grateful to $\mathrm{S}$. Machida for his interest.

\section{Appendix A. Normalization of Variables}

Prior to the numerical experiment, we need to obtain dimensionless equations. The variables are normalized by the following parameters: proton and electron number densities $n_{0}$, magnetic field $B_{0}$, electric field $E_{0}$, electric current $J_{0}$, velocity $u_{0}$, spatial scale $r_{0}$, time $t_{0}$, and the mass and the electric charge of an electron, $m_{0}$ and $q_{0}$, respectively.

In order that the equations of motion (1) remain the same after the normalization, we need the relationships

$$
\begin{aligned}
r_{0} & =u_{0} t_{0} \\
m_{0} u_{0} & =t_{0} q_{0} E_{0}
\end{aligned}
$$

and

$$
E_{0}=B_{0} .
$$

Normalizing the Gauss' law (6), we have

$$
E_{0}=n_{0} r_{0} q_{0}
$$

By substituting Eqs. (A.1) and (A.4) into (A.2) we obtain

$$
t_{0}=\omega_{p 0}^{-1} \equiv\left(\sqrt{\frac{n_{0} q_{0}^{2}}{m_{0}}}\right)^{-1}
$$

We choose $\omega_{p 0}$ the same as the plasma frequency of the unperturbed solar wind observed in the vicinity of the moon. That is, the time is normalized by the inverse plasma frequency, and the normalized plasma frequency is unity in the simulation scheme. Since the plasma frequency of the solar wind plasma was $18 \mathrm{kHz}$ at the time of GEOTAIL observation of the wake-related ULF waves (Nakagawa et al., $2003)$, the time scale $t_{0}$ corresponds to $8.8 \times 10^{-6}[\mathrm{~s}]$.

The factors for the mass and the charge of an electron $m_{0}$ and $q_{0}$ are chosen so that the normalized ratio $q / m$ would become unity. That is, the ratio $q_{0} / m_{0}$ is equal to the real value of $|q| / m_{e}$. Combined with Eq. (A.5), it leads to the choice of the factors for the number density $n_{0}$ and the electric charge $q_{0}$ so that the normalized value $n q$ should be unity.

In a 2-D simulation, the normalized number density $n=$ $n_{\text {real }} / n_{0}$ is given by the number of particles per area, not per volume. For example, the normalized number density $\langle n\rangle$ averaged over the simulation domain is $N_{p} / L^{2}$, thus the dimension of the normalizing factor $n_{0}=\left\langle n_{\text {real }}\right\rangle /\langle n\rangle=$ $\left\langle n_{\text {real }}\right\rangle L^{2} / N_{p}$ is not the same as that of the real density $\left[\mathrm{m}^{-3}\right]$. The situation is the same for the electric charge $q_{0}=|q| N_{p} / L^{2}$ and the electron mass $m_{0}=m_{e} N_{p} / L^{2}$, where $|q|$ and $m_{e}$ are the real values. These parameters appear only in the form of $n q$ and $m / q$ in the equations, giving no effect on the electric field or the potential.

In this paper, we use the electron thermal speed $v_{e}$ as the normalizing parameter $u_{0}$. Accordingly, the spatial scale becomes $r_{0}=v_{e} / \omega_{p 0}=\sqrt{2} \lambda_{D}$.

From Eq. (A.2) together with (A.3) and (A.5) we have

$$
E_{0}=B_{0}=\frac{m_{0}}{q_{0}} u_{0} \omega_{p 0},
$$

which corresponds to the electric field of $1.6 \mathrm{~V} / \mathrm{m}$ and the magnetic field of 5.3 nT in MKSA unit. It leads the normalizing factor for the electric potential $\phi_{0}=E_{0} t_{0}=m_{0} v_{e}^{2} / q_{0}$. It corresponds to $30-40 \mathrm{~V}$ when the electron thermal energy is $15-30 \mathrm{eV}$. 


\section{References}

Beers, B. L., Numerical calculation of the lunar wake in a magnetohydrodynamic model, Phys. Fluids, 15, 1450-1456, 1972.

Beers, B. L., Erratum: Numerical calculation of the lunar wake in a magnetohydrodynamic model, Phys. Fluids, 16, 456, 1973.

Birdsall, C. K. and A. B. Langdon, Plasma Physics via Computer Simulation, McGraw-Hill, New York, 1985.

Birch, P. C. and S. C. Chapman, Correction to "Particle-in-cell simulations of the lunar wake with high phase resolution", Geophys. Res. Lett., 28, 2669, 2001.

Birch, P. C. and S. C. Chapman, Two dimensional particle-in-cell simulations of the lunar wake, Phys. Plasmas, 9, 1785-1789, 2002.

Colburn, D. S., R. G. Currie, J. D. Mihalov, and C. P. Sonett, Diamagnetic solar-wind cavity discovered behind moon, Science, 158, 1040-1042, 1967.

Fahleson, U., Theory of electric field measurements conducted in the magnetosphere with electric probes, Space Sci. Rev., 7, 238-262, 1967.

Farrell, W. M., R. J. Fitzenreiter, C. J. Owen, J. B. Byrnes, R. P. Lepping, K. W. Ogilvie, and F. Neubauer, Upstream ULF waves and energetic electrons associated with the lunar wake: Detection of precursor activity, Geophys. Res. Lett., 23, 1271-1274, 1996.

Farrell, W. M., M. L. Kaiser, J. T. Steinberg, and S. D. Bale, A simple simulation of a plasma void: Applications to Wind observations of the lunar wake, J. Geophys. Res., 103, 23635-23653, 1998.

Futaana, Y., S. Machida, and T. Saito, A. Matsuoka, and H. Hayakawa, Counterstreaming electrons in the near vicinity of the moon observed by plasma instruments on board NOZOMI, J. Geophys. Res., 106, 1872918740, 2001.

Guio, P. and H. L. Pécseli, Phase space structures generated by an absorbing obstacle in a streaming plasma, Geophys. Res. Lett., 31, L03806, 2004.

Guio, P. and H. L. Pécseli, Phase space structures generated by absorbing obstacles in streaming plasmas, Ann. Geophys., 23, 853-865, 2005.

Halekas, J. S., D. L. Mitchell, R. P. Lin, L. L. Hood, M. N. Acunã, and A. B. Binder, Evidence for negative charging of the lunar surface in shadow, Geophys. Res. Lett., 29, 77, doi:10.1029/2001GL014428, 2002.

Halekas, J. S., R. P. Lin, and D. L. Mitchell, Inferring the scale height of the lunar nightside double layer, Geophys. Res. Lett., 30, PLA1, 2117, doi:10.1029/2003GL018421, 2003.

Halekas, J. S., S. D. Bale, D. L. Mitchell, and R. P. Lin, Electrons and mag- netic fields in the lunar plasma wake, J. Geophys. Res., 110, A07222, doi:10.1029/2004JA010991, 2005.

Kallio, E., Formation of the lunar wake in quasi-neutral hybrid model, Geophys. Res. Lett., 32, L06107, doi:10.1029/2004GL021989, 2005.

Kellogg, P. J., K. Goetz, and S. J. Monson, Observation of lunar plasma waves during a travel $\mathrm{f}$ the moon's wake, Geophys. Res. Lett., 23, 12671270, 1996.

Lipatov, A. S., Three dimensional structure of the plasma wake of the moon, Cosmic Res., 14, 103-106, 1976.

Lyon, E. F., H. S. Bridge, and J. H. Binsak, Exploreer 35 plasma measurements in the vicinity of the moon, J. Geophys. Res., 72, 6113, 1967.

Nakagawa, T. and M. Iizima, Pitch angle diffusion of electrons at the boundary of the lunar wake, Earth Planets Space, 57, 885-894, 2005.

Nakagawa, T. and M. Iizima, A reexamination of pitch angle diffusion of electrons at the boundary of the lunar wake, Earth Planets Space, 58, e17-e20, 2006.

Nakagawa, T., Y. Takahashi, and M. Iizima, GEOTAIL observation of upstream ULF waves associated with lunar wake, Earth Planets Space, 55, 569-580, 2003.

Ness, N. F., K. W. Behannon, H. E. Taylor, and Y. C. Whang, Perturbations of the interplanetary magnetic field by the lunar wake, J. Geophys. Res., 73, 3421-3440, 1968.

Ogilvie, K. W., J. T. Steinberg, R. T. Fitzenreiter, C. J. Owen, A. J. Lazarus, W. M. Farrell, and R. B. Torbert, Observation of the lunar plasma wake from the WIND spacecraft on December 27, 1994, Geophys. Res. Lett., 23, 1255-1258, 1996.

Samir, U., K. H. Wright, Jr., and N. H. Stone, The expansion of a plasma into a vacuum: Basic Phenomena and processes and applications to space plasma physics, Rev. Geophys. Space Sci., 21, 1631-1646, 1983.

Schubert, G. and B. R. Lichtenstein, Observations of moon-plasma interactions by orbital and surface experiments, Rev. Geophys. Space Phys., 12, 592-626, 1974.

Trávníček, P., P. Hellinger, D. Schriver, and S. D. Bale, Structure of the lunar wake: Two-dimensional global hybrid simulations, Geophys. Res. Lett., 32, L06102, doi:10.1029/2004GL022243, 2005.

Whang, Y. C., Field and plasma in the lunar wake, Phys. Rev., 186, $143-$ $150,1969$.

S. Kimura and T. Nakagawa (e-mail: nakagawa@tohtech.ac.jp) 